\title{
Assessment of Ankle Brachial Index among Stroke and Non- stroke patients in a Tertiary care hospital
}

\author{
Sanjay Polisetty ${ }^{1} \&$ Dr. Krishna Veni Avvaru ${ }^{2}$ \\ ${ }^{1} 3 r d$ year MBBS student, Andhra Medical College, Visakhapatnam \\ ${ }^{2}$ Professor of Community Medicine, Rajiv Gandhi Institute of Medical Sciences, Srikakulam
}

\begin{abstract}
:
Background: Stroke is an important cause of premature death and disability in developing countries such as India. A low ankle-brachial index (ABI) is predictive of peripheral arterial disease. Early assessment of the patient may be beneficial in rapid identification of severe cases, treatment and most importantly, disability limitation.

Objectives: 1.To estimate and compare ABI among stroke and non- stroke patients .2.To study association of ABI and NIHSS score among study population.

Methodology: A case control study was conducted among 50 patients of ischaemic stroke and 50 patients having no previous history of stroke. The study variables included Age, Gender, Ankle brachial index (ABI), NIHSS score etc. Data analysis was done using MS Excel and relevant statistical tests were applied. Results: Majority of the study participants were in the age groups of 51-70 years; $82 \%$ were males; $22 \%$ of stroke patients had low ABI and 64\% had normal ABI as compared to $82 \%$ of non-stroke patients; Mean NIHSS score was 22.5 among patients who had low ABI.
\end{abstract}

Conclusion: Normal range of Ankle brachial index was observed to be significantly higher among non-stroke patients as compared to stroke patients. Patients with low ABI values presented with severe stroke.

Keywords: Ischaemic Stroke, Ankle brachial index, NIHSS score, Peripheral artery disease.

\section{Introduction}

Stroke is becoming an important cause of premature death and disability in low-income and middleincome countries like India, largely driven by demographic changes and enhanced by the increasing prevalence of the key modifiable risk factors. [1] As a result, developing countries are exposed to a double burden of both communicable and non-communicable diseases. The estimated adjusted prevalence rate of stroke varies between $84-262 / 100,000$ in rural and 334-424/100,000 in urban areas [2]. The incidence rate is $119-145 / 100,000$ based on the recent population based studies [3]. Majority of stroke survivors continue to live with disabilities due to the lack of early detection.

Ankle brachial index (ABI), which is ratio of the systolic blood pressure of the Posterior Tibial artery to the systolic blood pressure of the brachial artery, is known to be a measure of lower limb peripheral artery disease [PAD] as well as a marker for other cardiovascular disease events. [4]. In middle-aged and older adults a low ABI has been reported to be associated with an increased risk of death, total cardiovascular disease (CVD), coronary heart disease (CHD), congestive heart failure, and symptomatic peripheral arterial disease[5]. Ankle brachial index of 0.8-0.9 indicate some arterial disease and 0.5 - 0.8 indicate moderate arterial disease. ABI under 0.5 indicates severe arterial disease and needs urgent specialist referral [6].

Many studies have been conducted to determine any possible relation between ABI and the initial severity of stroke as well as its outcome .ABI is a simple and non-invasive test that can not only be applied to diagnose PAD, but also to provide important prognostic information. This test is mostly underutilized by medical practitioners. Early assessment of the patient may be beneficial in rapid identification of severe cases, treatment and most importantly, disability limitation $[7,8]$.

Having stated the above findings, the present study has been taken up to estimate ABI among stroke patients and also to compare the ankle brachial index among stroke and non stroke patients attending a tertiary care hospital.

\section{Materials And Methods}

A case control study was conducted at the Medicine and Neuromedicine departments of King George Hospital, a tertiary care centre in Visakhapatnam, Andhra Pradesh. Data was collected for a period of 3 months from $1^{\text {st }}$ April 2014 to $30^{\text {th }}$ June, 2014.

A total of 50 patients admitted due to ischaemic stroke were included in the study. For the purpose of randomisation, every patient with an odd IP number was considered for the study. Comparison group includes 
50 patients admitted in the same wards who had no history of stroke or PAD. Age and Gender were matched. Informed consent was obtained from the study subjects. A pretested questionnaire was used for obtaining data from the patients. Systolic blood pressure of the posterior tibial and brachial artery was measured using an automated oscillometric blood pressure measurement device and the ankle brachial index was calculated.ABI was divided as $<0.9$ (abnormal low), 0.9-1.4(normal) and $>1.4$ (abnormal high). Severity of stroke was assessed by using the National Institute of Health Stroke Scale (NIHSS). The study variables were Age, Gender, NIHSS score etc.

All those patients from whom consent could not be obtained and whose blood pressure could not be measured were excluded from the study. Smokers and Diabetics were also excluded as these conditions are also responsible for low ABI. Hemorrhagic stroke cases were also not included in this study due to the emergency condition of those patients. Data analysis was done using MS Excel sheet and relevant tests of significance were applied. $\mathrm{p}<0.05$ was considered as statistically significant.

\section{Results}

Majority of the study population were males (82\%) and the rest $18 \%$ were females. The age of the study participants ranged from 31- 84 years. Majority (66\%) were in the age group of 51-70 years, $14 \%$ in the age group of 41-50 years and 2 patients were between 31-40 years of age. Among the stroke cases, $40 \%$ hailed from rural background and the rest hailed from urban areas. Of the test subjects, $17 \%$ of the cases had low BMI $(<18.5)$, while $47 \%$ of them had normal BMI (18.5-22.9) and the rest were either pre-obese or obese.

Table 1: Age-wise distribution of subjects in the study and control group

\begin{tabular}{|c|c|c|}
\hline Age in years & Study group & Control group \\
\hline $31-40$ & 2 & 2 \\
\hline $41-50$ & 7 & 7 \\
\hline $51-60$ & 15 & 15 \\
\hline $61-70$ & 18 & 18 \\
\hline$>71$ & 8 & 8 \\
\hline Total & 50 & 50 \\
\hline
\end{tabular}

Table 2: Ankle and brachial mean blood pressure levels among stroke and control group

\begin{tabular}{|l|c|c|c|c|}
\hline \multicolumn{1}{|c|}{ Blood pressure } & \multicolumn{2}{c|}{$\begin{array}{c}\text { Study group } \\
\text { Mean values(mm Hg) }\end{array}$} & \multicolumn{2}{c|}{$\begin{array}{c}\text { Control group } \\
\text { Mean values(mm Hg) }\end{array}$} \\
\hline & Systolic & Diastolic & Systolic & Diastolic \\
\hline Ankle & 126.7 & 92 & 131.3 & 99 \\
\hline Brachial & 120.4 & 83.2 & 122.6 & 84.3 \\
\hline
\end{tabular}

The Brachial systolic blood pressure ranged from $80-140 \mathrm{~mm} \mathrm{Hg}$ with a mean of $120.4 \mathrm{~mm} \mathrm{Hg}$ in the study group and 92-132 $\mathrm{mm} \mathrm{Hg}$ with a mean of $122.6 \mathrm{~mm} \mathrm{Hg}$ in the control group. Brachial diastolic blood pressure varied from $45-104 \mathrm{~mm} \mathrm{Hg}$ with a mean of $83.2 \mathrm{~mm} \mathrm{Hg}$ in the study group and 72-110 mm $\mathrm{Hg}$ with a mean of $84.3 \mathrm{~mm} \mathrm{Hg}$ in the control group.

Table 3: Comparison between ABI of the study and control groups

\begin{tabular}{|c|c|c|c|}
\hline Ankle brachial index(ABI) & Study group & Control group & $\mathrm{p}$ value \\
\hline$<0.9$ & $11(22 \%)$ & $5(10 \%)$ & $\mathrm{P}>0.05(\mathrm{Z}=1.66)$ \\
\hline $0.9-1.4$ & $32(64 \%)$ & $41(82 \%)$ & $\mathrm{P}<0.05(\mathrm{Z}=2.19)$ \\
\hline$>1.4$ & $7(14 \%)$ & $4(8 \%)$ & $\mathrm{P}>0.05(\mathrm{Z}=0.96)$ \\
\hline Total & $50(100 \%)$ & $50(100 \%)$ & \\
\hline
\end{tabular}

More than $1 / 5^{\text {th }}(22 \%)$ of stroke patients had low ABI $(<0.9)$ as compared to $10 \%$ in the control group. Normal ABI ( 0.9-1.4) was seen in $82 \%$ of the non-stroke patients as compared to $64 \%$ of stroke patients and this difference was found to be statistically significant $(\mathrm{z}=2.19$ and $\mathrm{p}<0.05)$.

Table 4: ABI vs. mean NIHSS stroke score in the stroke patients

\begin{tabular}{|c|c|}
\hline Ankle brachial index (ABI) & Mean NIHSS stroke score \\
\hline$<0.9$ & 22.5 \\
\hline $0.9-1.4$ & 8.3 \\
\hline$>1.4$ & 8 \\
\hline
\end{tabular}

In our study, among those who had ABI less than 0.9 , the mean NIHSS score was 22.5 indicating severe stroke whereas it was 8.3 among patients having normal ABI. 


\section{Discussion}

Most to the study population (60\%) belonged to urban background while $40 \%$ of the patients belonged to rural areas. The urban population had higher BMI in comparison with the rural population. This may be attributed to altering life style and lack of physical exercise [12]. . Males were higher in number than females indicating the male predominance of Stroke. $66 \%$ of the cases belonged to the 5th and 6th decade of life as in other studies [10].

The presence of abnormal ABI in ischaemic stroke patients was found to be $22 \%$. This finding is slightly higher than in previous studies conducted on ischaemic stroke patients [9]. In our study, patients with low ABI presented with more severe ischaemic stroke and this finding is in concurrence with Lee AJ et al, where they stated that low ABI was independently associated with higher NIHSS score[6]. Similarly Zheng et al observed that low ABI levels particularly of $<0.9$ are indicative of generalised atherosclerosis [5].

\section{Conclusion}

Normal range of Ankle brachial index was observed to be significantly higher among non-stroke patients as compared to stroke patients. Patients with low ABI values presented with severe stroke. Ankle brachial Index can easily be measured in a primary care setting and could be used as part of assessment of cardiovascular risk in individuals.

\section{References}

[1]. Jeyaraj Durai Pandian and Paulin SudhanStroke Epidemiology and Stroke Care Services in India.J Stroke. Sep 2013; 15(3): 128134.

[2]. Asian acute stroke advisory panel: stroke epidemiological data of nine Asian countries. J Med Assoc Thai. 2000; 83: 1-7.

[3]. Dalal PM. Studies in young and elderly: risk factors and strategies for stroke prevention. J Assoc Physicians India. 1997; 45: 125131.

[4]. Schroll M, Munck O. Estimation of peripheral arteriosclerotic disease by ankle blood pressure measurements in a population study of 60-year-old men and women. J Chronic Dis. 1981; 34: 261-269

[5]. Zheng ZJ, Sharrett AR, Chambless LE, Rosamond WD, Nieto FJ, Sheps DS, Dobs A, Evans GW, Heiss G. Associations of anklebrachial index with clinical coronary heart disease, stroke and preclinical carotid and popliteal atherosclerosis: the Atherosclerosis Risk in Communities (ARIC) Study. Atherosclerosis. 1997; 131: 115-125.

[6]. Leng GC, Fowkes FG, Lee AJ, Dunbar J, Housley E, Ruckley CV. Use of ankle brachial pressure index to predict cardiovascular events and death: a cohort study. BMJ. 1996; 313: 1440-1444.

[7]. Newman AB, Shemanski L, Manolio TA, Cushman M, Mittelmark M, Polak JF, Powe NR, Siscovick D. Ankle-arm index as a predictor of cardiovascular disease and mortality in the Cardiovascular Health Study. The Cardiovascular Health Study Group. Arterioscler Thromb Vasc Biol.1999; 19: 538-545.

[8]. Resnick HE, Lindsay RS, McDermott MM, et al. Relationship of high and low ankle brachial index to all-cause and cardiovascular disease mortality: the Strong Heart Study. Circulation. 2004;109:733-739

[9]. Joanne M. Murabito, MD, ScM; Jane C. Evans, DSc, The Ankle-Brachial Index in the Elderly and Risk of Stroke, Coronary Disease, and Death, Arch Intern Med. 2003;163(16):1939-1942.

[10]. Purroy F1, Coll B, Oró M, Setó E, Piñol-Ripoll G, Plana A, Quílez A, Sanahuja J, Brieva L, Vega L, Fernández E, Predictive value of ankle brachial index in patients with acute ischaemic stroke.Eur J Neurol. 2010 Apr;17(4):602-607

[11]. Tziomalos K, Giampatzis V, Bouziana S, Pavlidis A, Spanou M, Papadopoulou M, Kagelidis G, Boutari C, Savopoulos C, Hatzitolios A.Predictive value of the ankle brachial index in patients with acute ischemic stroke.Vasa. 2014 Jan;43(1):55-61

[12]. Tsivgoulis G1, Bogiatzi C, Heliopoulos I, Vadikolias K, Boutati E, Tsakaldimi S, Al-Attas OS, Charalampidis P, Piperidou C, Maltezos E, Papanas N.Low ankle-brachial index predicts early risk of recurrent stroke in patients with acute cerebral ischemia.Atherosclerosis. 2012 